\title{
Characteristics of Molecularly Imprinted Polymer Thin Layer for Bisphenol A and Response of the MIP-Modified Sensor
}

\author{
Izumi Kubo, ${ }^{1}$ Nobuyuki Yokota, ${ }^{1}$ Yusuke Fuchiwaki, ${ }^{2}$ and Yuko Nakane ${ }^{1,3}$ \\ ${ }^{1}$ Department of Bioinformatics, Graduate School of Engineering, Soka University, 1-236 Tangi, Hachioji, Tokyo 192-8577, Japan \\ ${ }^{2}$ Health Technology Research Center, National Institute of Advanced Industrial Science and Technology, 1-8-31 Midorigaoka, \\ Ikeda, Osaka 563-8577, Japan \\ ${ }^{3}$ RIKEN Quantitative Biology Center, 6-2-3 Furuedai, Suita, Osaka 565-0874, Japan \\ Correspondence should be addressed to Izumi Kubo, kubo@t.soka.ac.jp
}

Received 1 September 2011; Accepted 12 October 2011

Academic Editor: J. Gruber

Copyright ( $) 2012$ Izumi Kubo et al. This is an open access article distributed under the Creative Commons Attribution License, which permits unrestricted use, distribution, and reproduction in any medium, provided the original work is properly cited.

We examine the characteristics of molecularly imprinted polymer (MIP) layers for bisphenol A (BPA) to investigate the effect of their thickness on the performance of the BPA sensor. MIP thin layers for bisphenol A were polymerized on a sputtered gold electrode by UV light irradiation for 2 to $30 \mathrm{~min}$. Their thickness, as determined by a QCM analyzer, was $3.6 \pm 0.3 \mathrm{~nm}$ after $5 \mathrm{~min}$ of irradiation and increased as the irradiation time increased to $30 \mathrm{~min}$. AFM images of the MIP-modified surface suggested that the gold electrode was covered with a smooth MIP layer. The anodic peaks of BPA and ascorbic acid caused by gold electrode and the MIP-modified electrode were compared, and the electrode with MIP polymerized for 5 min showed more selectivity to BPA than that polymerized for $2 \mathrm{~min}$. The MIP thin layer thus has potential as a sensing element of a chemical sensor.

\section{Introduction}

Enormous amounts of bisphenol A (BPA) have been used as a major material to produce polycarbonate, which is widely used in food containers because of its heat durability and solidity. When hot food or drink is placed into the polycarbonate container, BPA remaining in the plastic tends to dissolve into the food or drink. The eluted BPA from the containers might be consumed with food into the human body. Evidence from laboratory studies suggests that BPA may act a toxicant for developing tissues and has stimulated debate regarding the impact of human exposure to BPA [1]. Animal studies suggest that prenatal and early postnatal exposure to BPA may cause serious effects on brain development [2-4], sexual differentiation [5], behavior [2, 6, 7], and immune function [8-10]. There is a fear that contamination of BPA in food or drink may have a broad range of adverse effects on public health [11-14]. In Japan the concentration in food or drink must be lower than $2.5 \mathrm{ppm}$ as established by food hygiene law. Conventionally, BPA concentration has been measured with the use of HPLC [15-18] or GC/MS $[19,20]$. These apparatuses are expensive and the methods need sample pretreatment. Thus, a rapid and easy method for monitoring and analyzing BPA is required.

We have been working on developing a simple and easy monitoring system for BPA based on an electrochemical reaction and artificial receptor of BPA. Since BPA is an electroactive species, it can be oxidized and detected electrochemically at $2.5 \mathrm{ppm}$ [21]. As a promising artificial receptor, we utilized molecularly imprinted polymer (MIP). MIP particles for BPA were thermally synthesized by Sanbe and Haginaka for the application to a column chromatography utilizing it as a specific binding resin [22]. MIP recognizes a template molecule in its recognition site and is expected to be more stable than biological receptor. We are working on utilizing MIPs as a recognition element of chemical sensors. We modified the preparation procedure of MIP for BPA reported by Sanbe and Haginaka and applied the fabricated MIP to detect BPA coupled with an electrochemical detection system. In this system MIP was utilized as small particles packed in a column [23]. We are also working on the establishment of a BPA sensor by fabricating an electrode with a layer of MIP, which has recognition sites for BPA. To detect BPA bound to MIP from a sample solution, 
oxidation of BPA is an efficient way, although MIP is itself an insulator and the redox reaction cannot be performed. In previous studies, we developed atrazine [24] and simazine $[25,26]$ sensors consisting of a gold electrode modified with an MIP layer synthesized by UV irradiation, and the sensor was immersed in $\mathrm{LiCl}$ solution to introduce an electrolyte into the MIP layer prior to redox reaction of these herbicides. Although this method was effective to measure redox current, the electrolyte could not be kept for long in the MIP layer [24]. Instead of introducing an electrolyte into the MIP layer, we decided to prepare an MIP layer on the surface of the electrode as thinly as possible by UV illumination to introduce BPA to the surface of the electrode.

A thin MIP layer is a promising element of chemical sensors [27-30] and its binding affinity and selectivity to analytes have been investigated. However, the effect of its thickness on the sensor response has not yet been reported.

In this study, we examine the characteristics of MIP layers to investigate the effect of their thickness on the performance of a BPA sensor. MIP was polymerized by UV irradiation, and we examined its irradiation time, thickness, and appearance, as well as the selectivity of the sensor.

\section{Experimental}

2.1. Materials and Methods. BPA, which is a standard chemical for environmental analysis, was obtained from Kanto Chemicals (Tokyo, Japan). 4-vinylpyridine (4-VPY), 2,2' azobis (2,4 dimethylvaleronitrile), and ethylene dimethacrylate (EDMA) were obtained from WAKO Pure Chemicals. Other reagents were of laboratory grade and used without any purification.

2.2. Preparation of MIP Receptor for BPA on QCM. In this study, we used a plate-type gold electrode (QA-A9-Au, Seiko EG\&G, Chiba, Japan) for the modification with MIP. As the shape of the electrode is a flat plate, it is suitable for direct polymerization of prepolymer solution on its surface. The size of the electrode is $5 \mathrm{~mm}$ in diameter.

Prior to preparing MIP layer on the electrode, a circular opening was fabricated on plate electrode with photosensitive polyimide insulator coating (Photoneece PW-1500 TORAY) to make defined area $(\phi=1.5 \mathrm{~mm})$ of electrochemical detection.

The surface of the gold layer was cleaned for $30-60 \mathrm{sec}$ by sputtering ionized air with an ion coater (IB-2, EIKO Engineering). Immediately after cleaning, the electrode was immersed in allyl mercaptan $(53 \mathrm{mM})$ for $2 \mathrm{~h}$ to introduce vinyl groups onto the surface of the electrode for covalent bonding to MIP, after which it was dried with nitrogen gas.

According to the slightly modified procedure reported previously [25], MIP was polymerized using BPA as template and 4-VPY and EDMA as a functional monomer and a cross-linker, respectively. A prepolymer cocktail was prepared by dissolving BPA (128 mg), 4-VPY (0.37 mL), EDMA $(2.5 \mathrm{~mL})$, and $2,2^{\prime}$-azobis (2,4 dimethylvaleronitrile) (30 mg) in toluene $(5 \mathrm{~mL})$. The prepolymer cocktail was purged with nitrogen gas for $3 \mathrm{~min}$ and spread on the electrode by spin coating at $1000 \mathrm{rpm}$ for $10 \mathrm{sec}$ with a spin-coater (Kyowa Riken, model K-359 S-1). Polymerization was performed by UV light $(350 \mathrm{~nm})$ irradiation with a hand-held UV lamp (Model UVGL-58, FUNAKOSHI, Tokyo) at room temperature. After irradiation, the MIP-modified electrode was dried at room temperature overnight. It was then washed in methanol repeatedly to remove unreacted reagents including BPA until no reagents were left in the methanol used in washing, as detected by UV absorption.

2.3. Measurement of the Thickness Apparatus of QCA. Quartz Crystal Microbalance (QCM) measurements were carried out with a Quartz Crystal Analyzer QCA 917 (SEIKO EG\&G) equipped with a $9 \mathrm{MHz}$ AT-Cut Au-QCM (Figure 1(a)) modified by an MIP thin layer. To determine the thickness of the MIP, before and after the polymerization of the MIP on the QCM electrode, the frequency shift was measured by the QCA. At first, the frequency shift of the naked QCM was measured. After the fabricating the dried MIP layer through the irradiation of UV light on the prepolymer cocktail on the electrode, the frequency shift decreased, and after washing off excess reagents, the frequency shift gradually increased and reached a steady state. The difference in the frequency shift between the naked QCM and the completely washed MIP layer-modified QCM was determined as mass of MIP.

The increase in the mass of $1 \mathrm{ng}$ on the surface of the QCM was determined as a decrease of $1 \mathrm{~Hz}$ by the QCA. The density of the MIP layer was estimated to be 1.0. From the density and the mass determined by the QCA, the volume of the MIP layer on the QCM was obtained. The thickness of the MIP was calculated through the division of the volume by the area of QCM electrode.

\subsection{Electrochemical Measurement. Electrochemical mea-} surements were performed with a voltammetry analyzer (ALS1200, BAS, Tokyo, Japan) and a three-electrode electrochemical cell. The MIP receptor-modified electrodes were used as the working electrode. $\mathrm{An} \mathrm{Ag} / \mathrm{AgCl}$ electrode and a platinum wire electrode were used as a reference and a counter electrode, respectively. All the electrochemical measurements were carried out in a glass vial containing $10 \mathrm{~mL}$ of deoxygenated solution $(0.1 \mathrm{M}$ phosphate buffer, $50 \mathrm{mM} \mathrm{KCl}$, $\mathrm{pH}$ 7.0) at room temperature.

2.5. AFM Observation. Topographic images were obtained by AFM to confirm the polymerized MIP on the electrode surface. AFM observations were carried out with a scanning probe microscope SPA-400 and probe station SPI-4000 (SII NanoTechnology Inc.) with a contact mode in the atmosphere.

The intrinsic resonance frequency of the cantilever was $12 \mathrm{kHz}$, and the spring constant was $0.16 \mathrm{~N} / \mathrm{m}$. Images were collected with a scan rate of $6 \mathrm{~Hz}$ for a scan area of $200 \mathrm{~nm}$. 


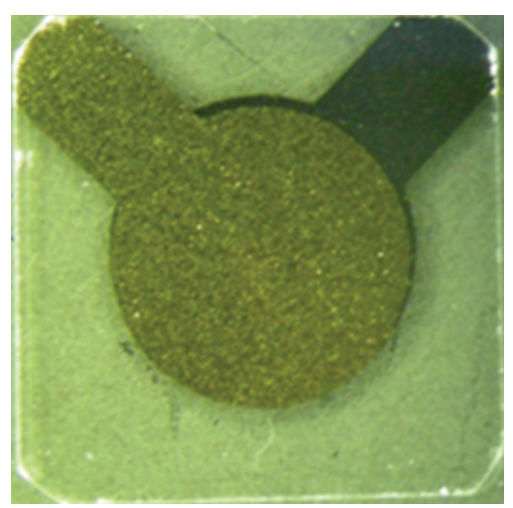

(a)

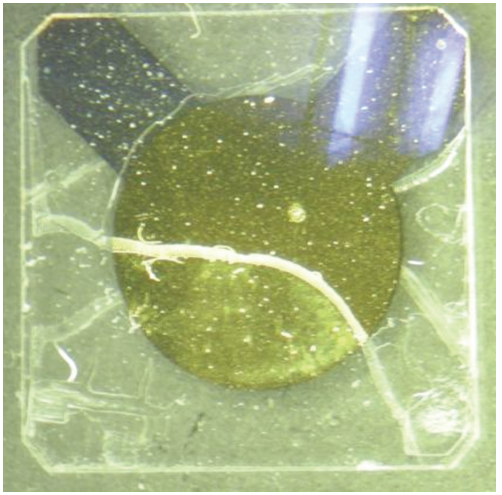

(b)

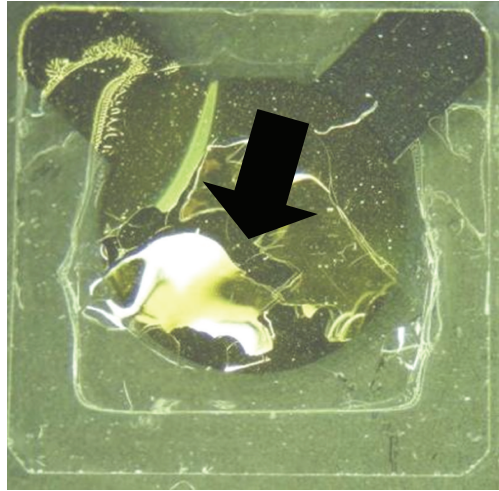

(c)

FIGURE 1: Photographs of BPA-MIP modified electrodes. (a) QCM electrode before MIP preparation. (b) The electrode after polymerization of MIP by UV irradiation for $2 \mathrm{~min}$. (c) The electrode after polymerization of MIP by UV irradiation for 30 min. The black arrow indicates a crack in the MIP film.

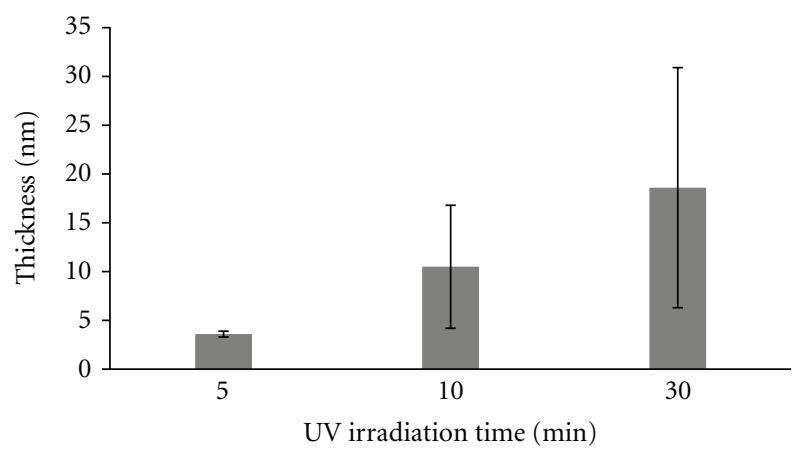

FIGURE 2: Thickness of the MIP film determined by QCM measurement and UV irradiation time.

\section{Results and Discussion}

3.1. Thickness of MIP. After irradiation of UV light for 2 to $30 \mathrm{~min}$, an MIP layer formed on the QCM electrode at a glance (Figures 1(b) and 1(c)). The thickness of the MIP layer on the electrode was determined by QCA at an irradiation time of 2, 5, 10, and $30 \mathrm{~min}$. At every irradiation time, three or more samples were prepared and served to QCM determination. Although the appearance of the MIP layer prepared by $2 \mathrm{~min}$ irradiation was rougher than the naked electrode, a decrease in frequency shift was not clearly observed. The thickness of the MIP layer increased as the irradiation time increased from 5 to $30 \mathrm{~min}$, and that of 5, 10 , and $30 \mathrm{~min}$ was $3.6 \pm 0.3,10.5 \pm 6.3$, and $18.6 \pm 12.3 \mathrm{~nm}$, respectively (Figure 2). A linear relationship was observed between irradiation time $(x, \mathrm{~min})$ and the thickness $(y, \mathrm{~nm})$ of the MIP layer: $y=0.54 x+2.4$. The thickness of these layers was in the nm range and the very thin layer of MIP was prepared by a short period of light irradiation. The thickness of the MIP layer polymerized after $5 \mathrm{~min}$ irradiation was the most reproducible among all irradiation times. Longer irradiation created a thicker layer and some of the MIP layer prepared after $30 \mathrm{~min}$ had cracks due to its rigidity (Figure 1(c)).

QCA analysis demonstrated that thin MIP layers were prepared through UV irradiation even only after $5 \mathrm{~min}$. However, the thickness is the value over the entire electrode on which MIP layer was prepared.

3.2. AFM Observation. AFM observations were performed to explore the finer structure of the MIP on the electrode. The topographic image of the surface of the gold electrode used in this study revealed that the electrode consisted of small gold grains (Figure 3(a)). The cross-sectional profile showed that the height and diameter of the grain was 6$7 \mathrm{~nm}$ and $80 \mathrm{~nm}$, respectively. It resembled a considerably flat hill with a slight slope. Then the MIPs prepared for 2, 5, and $10 \mathrm{~min}$ UV irradiation were observed. Prior to washes with methanol, the surface of the MIP layer polymerized for 10 min UV irradiation was completely different from the gold grain surfaces. No grain-like structure was observed and the surface was smoother than that of the gold electrode. After the washes with methanol, rougher surfaces appeared. The image before and after the washes was different between the naked gold grain and the smooth surface. As shown in Figure 3(b) the prepared MIP polymer covered the grains of sputtered gold and the surface became smoother. As for the MIPs prepared after 5 and 2 min UV irradiation, characteristic images were almost the same, although a distinct topographic image was not observed. The polymer was not considered to have formed grains but rather a flat layer along the grains (Figure 4). We then estimated the averaged slope angle from the cross-section profile of the AFM images and compared the change of the angles. The slope of the naked gold electrode was $4^{\circ}$ and after the polymerization but before washes, the angle decreased $1.5^{\circ}$ $(5 \mathrm{~min})$ and $2.0^{\circ}(2 \mathrm{~min})$. Then the slope increased again after washes to $2.4^{\circ}(5 \mathrm{~min})$ and $3.0^{\circ}(2 \mathrm{~min})$ (Figure 5). Even after 2 min irradiation, the surface was smoother than that of the naked gold electrode. 


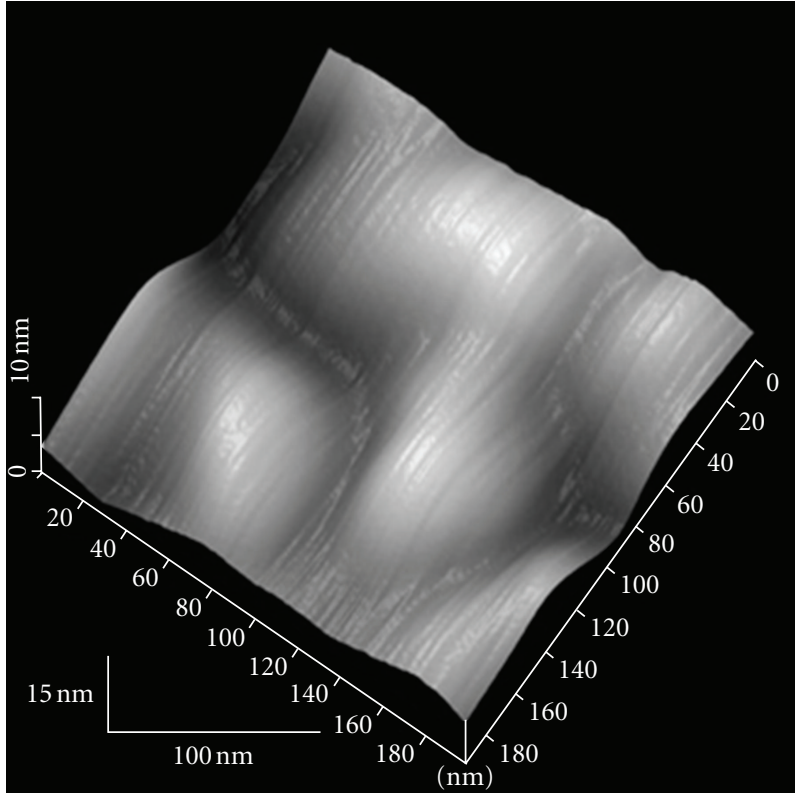

(a)

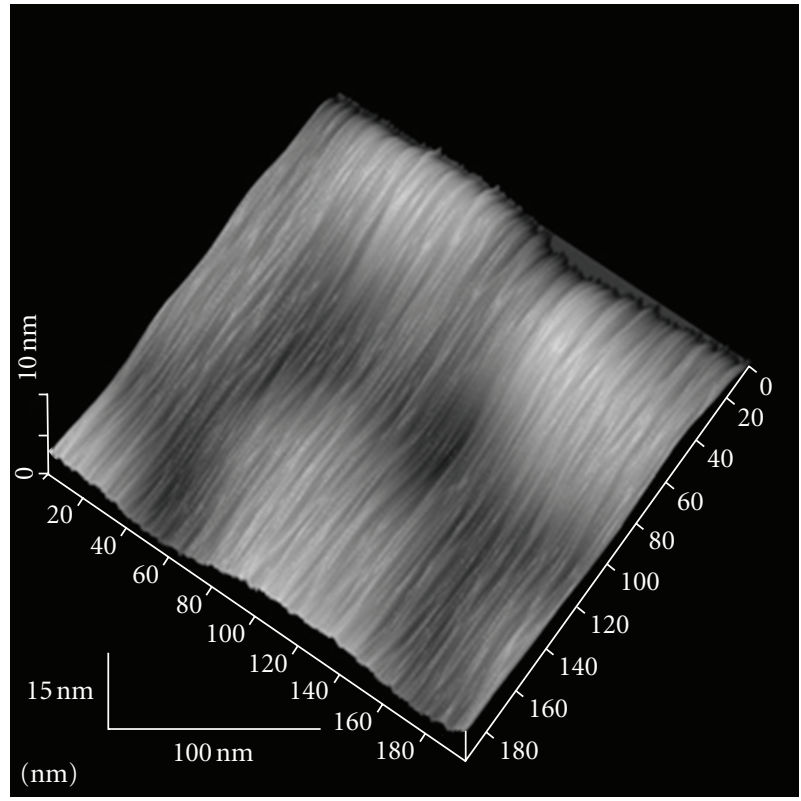

(b)

FIGURE 3: AFM images of the surface of bare electrode and MIP-electrode. (a) Bare electrode. (b) MIP-electrode polymerized for 5 min.

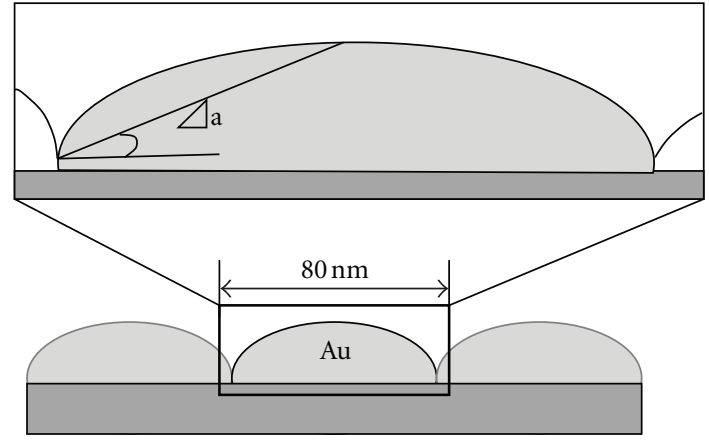

Figure 4: The concept of mean slope angle. Mean slope angle (triangle a) was calculated from the highest and lowest surface points in the arbitrary $80 \mathrm{~nm}$ area.

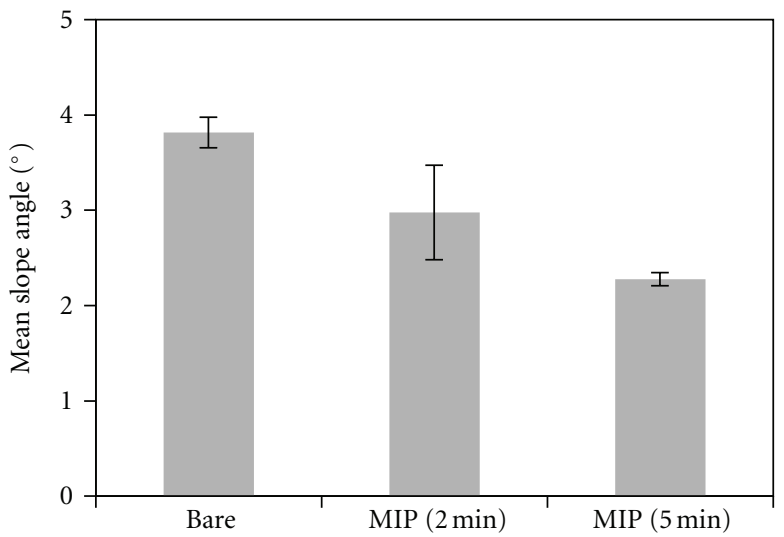

Figure 5: Polymerization time and mean slope angle after washes. The mean slope angles of the bare electrode and the MIP-electrode calculated by AFM image, $n=3$.
3.3. Electrochemical Response of the MIP-Modified Electrode. The electrochemical responses to BPA were examined with the use of an MIP-modified electrode. A cyclic voltammogram of ferricyanide $(5 \mathrm{mM})$ was examined to verify the coverage of the gold electrode by MIP. The electrode with MIP showed no redox peak for ferricyanide, irrespective of the irradiation time, while the bare gold electrode clearly showed a redox peak. As for the MIP prepared by $10 \mathrm{~min}$ UV irradiation, an electrochemical response to BPA was not observed with the use of the MIP-modified electrode; however, as for the electrodes prepared after 2 and $5 \mathrm{~min}$ irradiation, a response was clearly observed. The thickness of the MIP following 10 min UV irradiation might not be thin enough to obtain an electrochemical response. The MIPs prepared after 5 and 2 min UV irradiation were examined in detail and both of the MIP-modified electrodes showed an anodic peak current to BPA $(5 \mu \mathrm{M})$ at $580 \mathrm{mV}$ (Figure 6). The anodic peak potential to BPA observed with the use of the MIP thin-layer-modified electrode was the same as that of the naked gold electrode reported previously [23]. Then we compared the electrochemical response of the MIP thinlayer-modified electrode. An electroactive chemical species other than BPA, ascorbic acid, was examined, because food or biological samples often contain ascorbic acid, which may act as interference to the electrochemical measurement of BPA. Ascorbic acid was oxidized with the use of the naked gold electrode, and the peak potentials were $180 \mathrm{mV}$. With the use of the MIP-modified electrode, the anodic peak potential of BPA did not changed and the same with the MIP layer prepared for $2 \mathrm{~min}$ and 5 min UV irradiation, whereas the peak potential of ascorbic acid shifted $20 \mathrm{mV}$ to a positive potential. The thin MIP layer on the electrode may have worked as a blocking layer to the oxidation of 
TABLE 1: The response of the MIP-electrode and bare one to BPA and ascorbic acid.

\begin{tabular}{|c|c|c|c|c|c|}
\hline \multirow{2}{*}{ Electrode } & \multirow{2}{*}{ Polymerization } & \multicolumn{2}{|c|}{ BPA } & \multicolumn{2}{|c|}{ Ascorbic acid } \\
\hline & & $\begin{array}{l}\text { Peak potential } \\
\mathrm{mV}\end{array}$ & $\begin{array}{c}\text { Peak current } \\
\mathrm{nA} / \mu \mathrm{M}\end{array}$ & $\begin{array}{l}\text { Peak potential } \\
\mathrm{mV}\end{array}$ & $\begin{array}{c}\text { Peak current } \\
\mathrm{nA} / \mu \mathrm{M}\end{array}$ \\
\hline$\overline{\mathrm{Au}}$ & & 580 & $27.3(100 \%)$ & 180 & $13.6(100 \%)$ \\
\hline MIP & $2 \mathrm{~min}$ & 580 & $17.8(65.0 \%)$ & 200 & $7.6(55.9 \%)$ \\
\hline MIP & $5 \mathrm{~min}$ & 580 & $12.5(45.7 \%)$ & 200 & $4.5(32.9 \%)$ \\
\hline
\end{tabular}

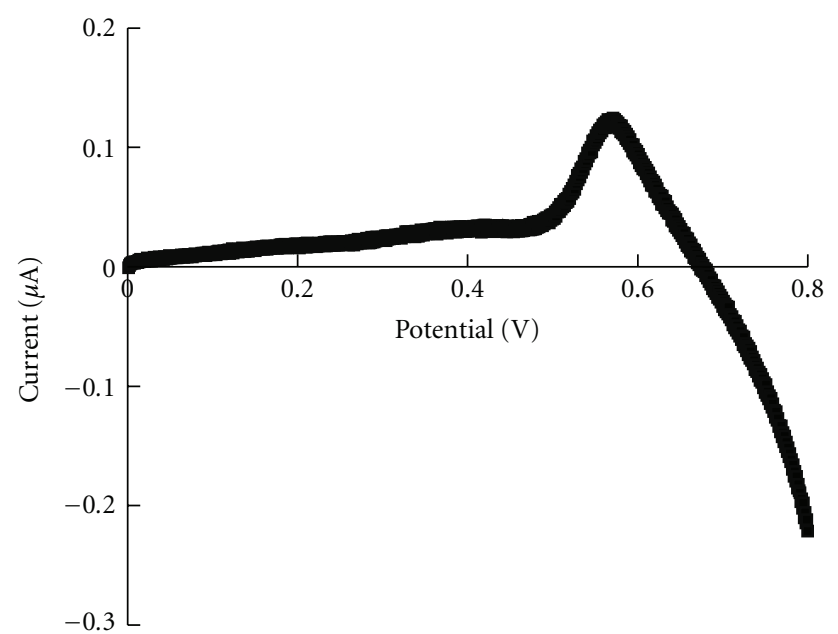

Figure 6: Cyclic voltammogram of BPA with MIP-modified electrode. Polymerization time of MIP was $5 \mathrm{~min}$. BPA concentration was $5 \mu \mathrm{M}$. Scan rate was $0.1 \mathrm{~V} / \mathrm{s} .0 .1 \mathrm{M}$ PBS, $\mathrm{pH}=7.0$.

ascorbic acid not only during electron transfer but also during the diffusion of ascorbic acid but not as a blocking layer to BPA because there are binding cites of BPA in the MIP layer. When we compared the anodic peak current of ascorbic acid and BPA, the currents to both decreased. The ratio of the current to that of the naked gold electrode, an index of the effect of the MIP layer to electron transfer, was much smaller for ascorbic acid than for BPA (Table 1). These phenomena demonstrated that BPA diffused to the MIP layer more selectively than ascorbic acid. Moreover, the MIP layer prepared after 5 min UV irradiation showed more selectivity than that prepared with 2 min irradiation, although the layer did not completely block the oxidation of ascorbic acid. The MIP layer did not work perfectly to block the diffusion of smaller molecules such as ascorbic acid $(\mathrm{Mw}=176.12)$, but its peak potential was small enough not to affect the electrochemical determination of BPA. Larger or hydrophobic molecules did not effect to the electrochemical reaction of BPA $(\mathrm{Mw}=228.29)$ when MIP-modified electrode was used.

In conclusion, a very thin layer of MIP was prepared by short UV irradiation to the prepolymer cocktail on the electrode and without introducing electrolyte into the layer, the MIP thin-layer-modified electrode showed an electrochemical response to BPA indicating that it has the potential as a sensing element of a chemical sensor.

\section{Acknowledgments}

This paper was supported in part by a Grant-in-Aids for Scientific Research (C) from the Ministry of Education, Culture, Sports, Science, and Technology (MEXT) Japan, and in part by funding from MEXT, the Matching Fund for Private Universities, S1001013, 2010-2015.

\section{References}

[1] C. A. Richter, L. S. Birnbaum, F. Farabollini et al., "In vivo effects of bisphenol A in laboratory rodent studies," Reproductive Toxicology, vol. 24, no. 2, pp. 199-224, 2007.

[2] K. Kubo, O. Arai, M. Omura, R. Watanabe, R. Ogata, and S. Aou, "Low dose effects of bisphenol A on sexual differentiation of the brain and behavior in rats," Neuroscience Research, vol. 45, no. 3, pp. 345-356, 2003.

[3] B. S. Rubin, J. R. Lenkowski, C. M. Schaeberle, L. N. Vandenberg, P. M. Ronsheim, and A. M. Soto, "Evidence of altered brain sexual differentiation in mice exposed perinatally to low, environmentally relevant levels of bisphenol A," Endocrinology, vol. 147, no. 8, pp. 3681-3691, 2006.

[4] S. Tando, K. Itoh, T. Yaoi, J. Ikeda, Y. Fujiwara, and S. Fushiki, "Effects of pre- and neonatal exposure to bisphenol A on murine brain development," Brain and Development, vol. 29, no. 6, pp. 352-356, 2007.

[5] S. Porrini, V. Belloni, D. D. Seta, F. Farabollini, G. Giannelli, and F. Dessì-Fulgheri, "Early exposure to a low dose of bisphenol A affects socio-sexual behavior of juvenile female rats," Brain Research Bulletin, vol. 65, no. 3, pp. 261-266, 2005.

[6] F. Dessì-Fulgheri, S. Porrini, and F. Farabollini, "Effects of perinatal exposure to bisphenol A on play behavior of female and male juvenile rats," Environmental Health Perspectives, vol. 110, supplement 3, pp. 403-407, 2002.

[7] K. Kawai, T. Nozaki, H. Nishikata, S. Aou, M. Takii, and C. Kubo, "Aggressive behavior and serum testosterone concentration during the maturation process of male mice: the effects of fetal exposure to bisphenol A," Environmental Health Perspectives, vol. 111, no. 2, pp. 175-178, 2003.

[8] M. Alizadeh, F. Ota, K. Hosoi, M. Kato, T. Sakai, and M. A. Satter, "Altered allergic cytokine and antibody response in mice treated with Bisphenol A," Journal of Medical Investigation, vol. 53, no. 1-2, pp. 70-80, 2006.

[9] M. Goto, Y. Takano-Ishikawa, H. Ono, M. Yoshida, K. Yamaki, and H. Shinmoto, "Orally administered bisphenol A disturbed antigen specific immunoresponses in the naïve condition," Bioscience, Biotechnology and Biochemistry, vol. 71, no. 9, pp. 2136-2143, 2007.

[10] H. Yan, M. Takamoto, and K. Sugane, "Exposure to bisphenol A prenatally or in adulthood promotes $\mathrm{TH} 2$ cytokine production associated with reduction of $\mathrm{CD} 4+\mathrm{CD} 25+$ regulatory $\mathrm{T}$ 
cells," Environmental Health Perspectives, vol. 116, no. 4, pp. 514-519, 2008.

[11] Y. Ikezuki, O. Tsutsumi, Y. Takai, Y. Kamei, and Y. Taketani, "Determination of bisphenol A concentrations in human biological fluids reveals significant early prenatal exposure," Human Reproduction, vol. 17, no. 11, pp. 2839-2841, 2002.

[12] S. Takayanagi, T. Tokunaga, X. Liu, H. Okada, A. Matsushima, and Y. Shimohigashi, "Endocrine disruptor bisphenol A strongly binds to human estrogen-related receptor $\gamma(\mathrm{ERR} \gamma)$ with high constitutive activity," Toxicology Letters, vol. 167, no. 2, pp. 95-105, 2006.

[13] J. M. Braun, K. Yolton, K. N. Dietrich et al., "Prenatal bisphenol A exposure and early childhood behavior," Environmental Health Perspectives, vol. 117, no. 12, pp. 1945-1952, 2009.

[14] L. F. Doherty, J. G. Bromer, Y. Zhou, T. S. Aldad, and H. S. Taylor, "In utero exposure to diethylstilbestrol (DES) or bisphenol-A (BPA) increases EZH2 expression in the mammary gland: an epigenetic mechanism linking endocrine disruptors to breast cancer," Hormones and Cancer, vol. 1, no. 3, pp. 146-155, 2010.

[15] J. E. Biles, K. D. White, T. P. McNeal, and T. H. Begley, "Determination of the diglycidyl ether of bisphenol A and its derivatives in canned foods," Journal of Agricultural and Food Chemistry, vol. 47, no. 5, pp. 1965-1969, 1999.

[16] R. Pulgar, M. F. Olea-Serrano, A. Novillo-Fertrell et al., "Determination of bisphenol A and related aromatic compounds released from Bis-GMA-based composites and sealants by high performance liquid chromatography," Environmental Health Perspectives, vol. 108, no. 1, pp. 21-27, 2000.

[17] J. Sajiki, K. Takahashi, and J. Yonekubo, "Sensitive method for the determination of bisphenol-A in serum using two systems of high-performance liquid chromatography," Journal of Chromatography B, vol. 736, no. 1-2, pp. 255-261, 1999.

[18] Y. Sun, M. Irie, N. Kishikawa, M. Wada, N. Kuroda, and K. Nakashima, "Determination of bisphenol A in human breast milk by HPLC with column-switching and fluorescence detection," Biomedical Chromatography, vol. 18, no. 8, pp. 501-507, 2004.

[19] S. Moors, M. Blaszkewicz, H. M. Bolt, and G. H. Degen, "Simultaneous determination of daidzein, equol, genistein and bisphenol A in human urine by a fast and simple method using SPE and GC-MS," Molecular Nutrition and Food Research, vol. 51, no. 7, pp. 787-798, 2007.

[20] N. Rastkari, R. Ahmadkhaniha, M. Yunesiana, L. J. Baleh, and A. Mesdaghiniaa, "Sensitive determination of bisphenol a and bisphenol $\mathrm{f}$ in canned food using a solid-phase microextraction fibre coated with single-walled carbon nanotubes before gc/ms," Food Additives and Contaminants, vol. 27, no. 10, pp. 1460-1468, 2010.

[21] H. Kuramitz, Y. Nakata, M. Kawasaki, and S. Tanaka, "Electrochemical oxidation of bisphenol A. Application to the removal of bisphenol A using a carbon fiber electrode," Chemosphere, vol. 45 , no. 1 , pp. 37-43, 2001.

[22] H. Sanbe and J. Haginaka, "Uniformly sized molecularly imprinted polymers for bisphenol A and $\beta$-estradiol: retention and molecular recognition properties in hydro-organic mobile phases," Journal of Pharmaceutical and Biomedical Analysis, vol. 30, no. 6, pp. 1835-1844, 2003.

[23] I. Kubo, N. Yokota, Y. Nakane, and Y. Fuchiwaki, "The establishment of bisphenol A (BPA) sensing system utilizing molecularly imprinted polymer receptor and electrochemical determination," International Journal of Electrochemistry, vol. 2011, Article ID 534936, 4 pages, 2011.
[24] R. Shoji, T. Takeuchi, and I. Kubo, "Atrazine sensor based on molecularly imprinted polymer-modified gold electrode," Analytical Chemistry, vol. 75, no. 18, pp. 4882-4886, 2003.

[25] Y. Fuchiwaki, R. Shoji, I. Kubo, and H. Suzuki, "6-ChloroN,N-diethyl-1,3,5-triazine-2,4-diamine (simazine) electrochemical sensing chip based on biomimetic recognition utilizing a molecularly imprinted polymer layer on a gold chip," Analytical Letters, vol. 41, no. 8, pp. 1398-1407, 2008.

[26] Y. Fuchiwaki, N. Sasaki, and I. Kubo, "Development of an electrochemical sensing system for 6-chloro-n,n-diethyl- 1,3,5triazine-2,4-diamine (CAT) utilizing an amalgamated gold electrode and artificial sensor receptor," Electrochemistry, vol. 75, no. 9, pp. 709-714, 2007.

[27] J. Matsui, Y. Miyoshi, O. Doblhoff-Dier, and T. Takeuchi, "A molecularly imprinted synthetic polymer receptor selective for atrazine," Analytical Chemistry, vol. 67, no. 23, pp. 4404-4408, 1995.

[28] T. A. Sergeyeva, S. A. Piletsky, A. A. Brovko, E. A. Slinchenko, L. M. Sergeeva, and A. V. El'skaya, "Selective recognition of atrazine by molecularly imprinted polymer membranes. Development of conductometric sensor for herbicides detection," Analytica Chimica Acta, vol. 392, no. 2-3, pp. 105-111, 1999.

[29] C. Luo, M. Liu, Y. Mo, J. Qu, and Y. Feng, “Thickness-shear mode acoustic sensor for atrazine using molecularly imprinted polymer as recognition element," Analytica Chimica Acta, vol. 428, no. 1, pp. 143-148, 2001.

[30] T. Panasyuk-Delaney, V. M. Mirsky, M. Ulbricht, and O. S. Wolfbeis, "Impedometric herbicide chemosensors based on molecularly imprinted polymers," Analytica Chimica Acta, vol. 435, no. 1, pp. 157-162, 2001. 

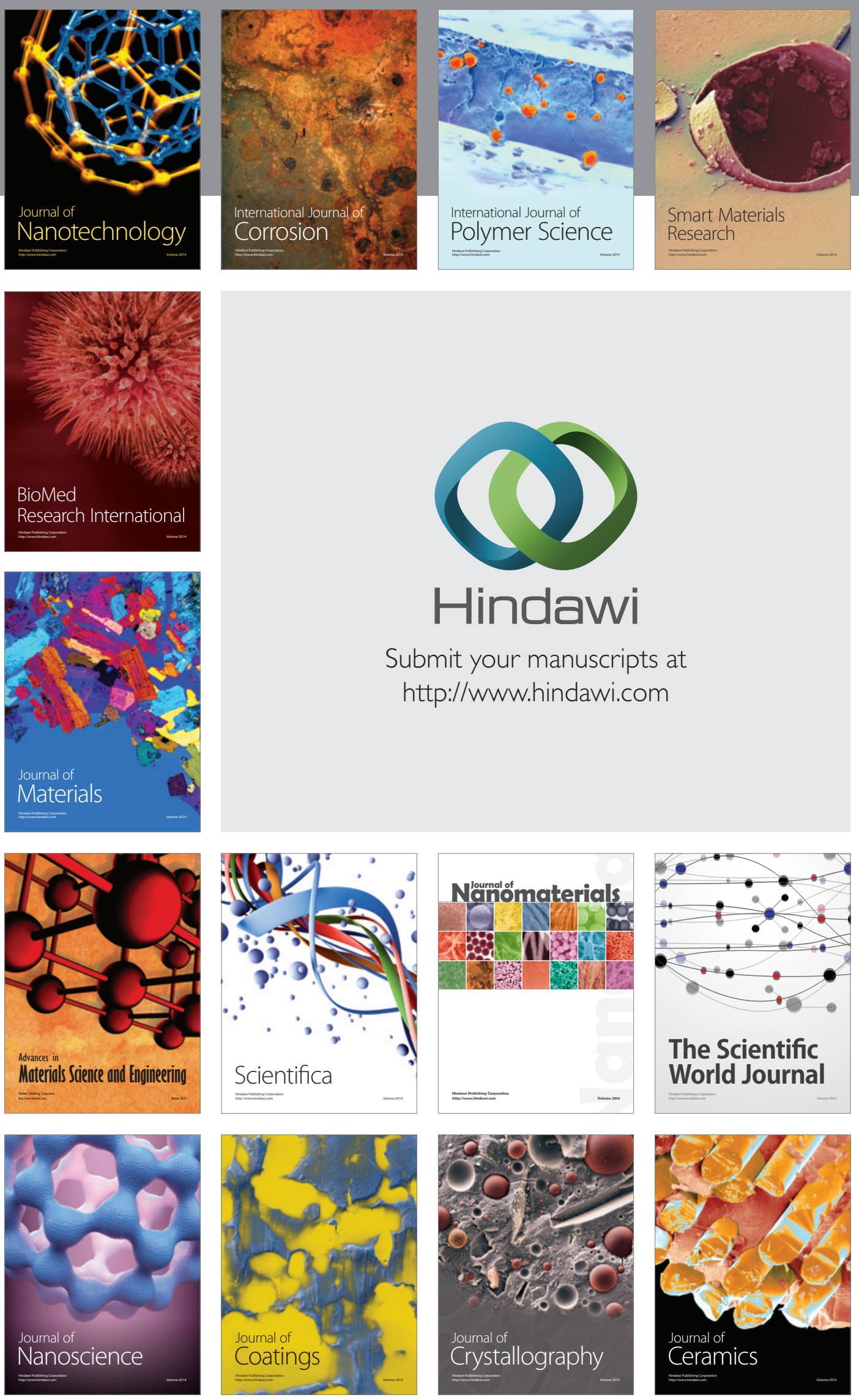

The Scientific World Journal

Submit your manuscripts at

http://www.hindawi.com

\section{World Journal}

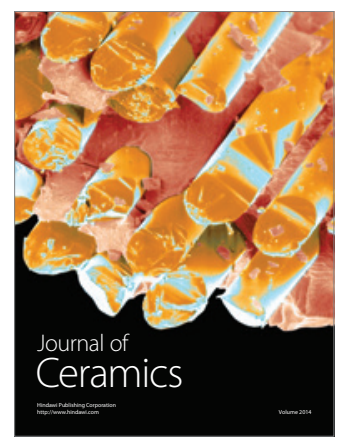

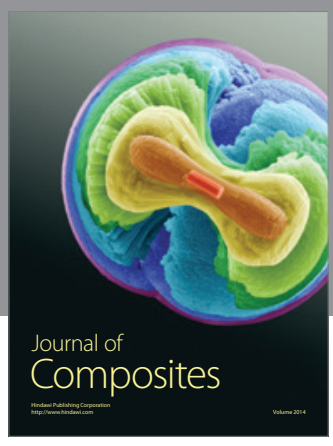
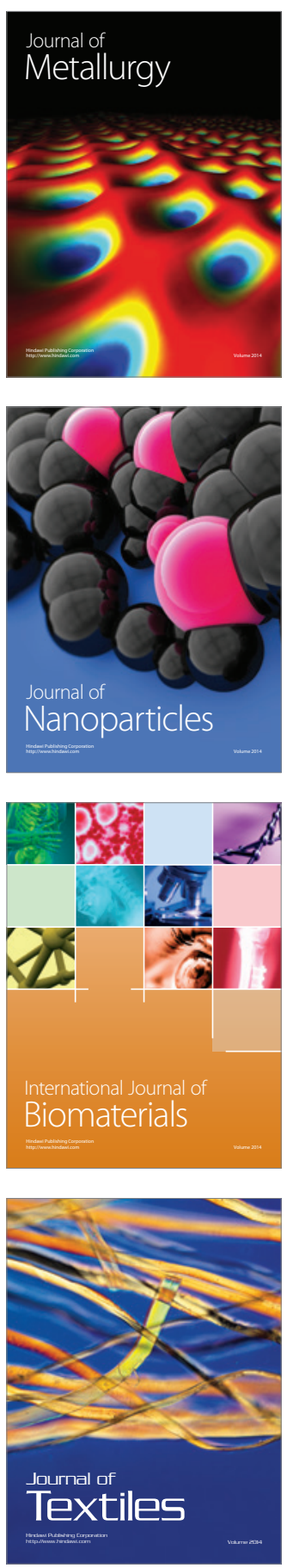